\title{
Predominant intragenic methylation is associated with gene expression characteristics in a bivalve mollusc
}

Characterization of DNA methylation patterns in the Pacific oyster, Crassostrea gigas, indicates that this epigenetic mechanism plays an important functional role in gene regulation and may be involved in the regulation of developmental processes and environmental responses. However, previous studies have been limited to in silico analyses or characterization of DNA methylation at the single gene level. Here, we have employed a genome-wide approach to gain insight into how DNA methylation supports the regulation of the genome in $C$. gigas. Using a combination of methylation enrichment and high-throughput bisulfite sequencing, we have been able to map methylation at over 2.5 million individual $\mathrm{CpG}$ loci. This is the first high-resolution methylome generated for a molluscan species. Results indicate that methylation varies spatially across the genome with a majority of the methylated sites mapping to intra-genic regions. The bisulfite sequencing data was combined with RNA-seq data to examine genome-wide relationships between gene body methylation and gene expression, where it was shown that methylated genes are associated with high transcript abundance and low variation in expression between tissue types. The combined data suggest DNA methylation plays a complex role in regulating genome activity in bivalves. 
1 Mackenzie R Gavery, Steven B Roberts ${ }^{\S}$

3 School of Aquatic and Fishery Sciences, University of Washington, 1122 NE Boat

4 Street, Seattle, Washington, USA

$5 \quad{ }^{\S}$ Corresponding author

6 Email addresses:

$7 \quad$ MRG: mgavery@u.washington.edu

$8 \quad$ SBR: sr320@u.washington.edu 


\section{Introduction}

10

Epigenetic marks such as DNA methylation are important for genome regulation (Bell \&

Felsenfeld 1992; Li et al., 1992; Hsieh 1994). DNA methylation has been well-studied in mammals and plants where it has been shown to play important roles in temporal and spatial regulation of gene expression (Okano et al.,1999, Zhang et al., 2006), reduction of transcriptional noise (Bird 1995), and genome stabilization (Wolffe \& Matzke 1999). However, the distribution and context of DNA methylation varies greatly among phylogenetic groups indicating that these functional roles may not be conserved (Colot \& Rossignol 1999).

In contrast to the heavily methylated vertebrate genomes, invertebrate genomes exhibit a relatively low level of methylation that is primarily confined to gene bodies (Zemach et al., 2010). It is perhaps because of these differences that DNA methylation has remained largely understudied in invertebrates. Recently, however, there has been a renewed interest in invertebrate DNA methylation patterns as it is now being recognized that they are exceptional models to study function and evolutionary roles of gene body methylation. Furthermore, it has been shown that DNA methylation mediates phenotypes in response to environmental cues in the invertebrate Apis mellifera (Kucharski et al., 2008, Lyko et al., 2010), indicating an important role integrating environmental signals. Thus, understanding both the distribution and function of DNA methylation in diverse invertebrate lineages will contribute significantly to our understanding of the evolution of genome regulation and environmental physiology.

The focus of the work presented here is to explore the role of DNA methylation in bivalve molluscs. The presence of DNA methylation has been confirmed in several bivalve species (Wang et al., 2008, Petrovic et al., 2009, Gavery \& Roberts 2010). A majority of the research on DNA methylation in molluscs has focused on the Pacific oyster (Crassostrea gigas), an economically and ecologically important species. Previous studies in the Pacific oyster identified 
33 a relationship between gene function and methylation pattern. Specifically, it was shown that

34 genes with housekeeping functions are more methylated than genes involved in inducible

35 functions (i.e. genes involved in response to environment, embryonic development or tissue-

36 specific functions) (Gavery \& Roberts 2010, Roberts \& Gavery 2012). More recently, Riviere et

37 al. (2013) determined that DNA methylation plays a critical role in development as indicated by

38 differential methylation patterns throughout embryogenesis. This was further supported by their

39 observation that 5-aza-cytidine, a potent demethylating agent, significantly disrupts embryonic

40 development (Riviere et al., 2013).

41 These recent studies on DNA methylation in oysters provides important foundational

42 information on DNA methylation in bivalves. However, previous studies were not able to

43 provide fine scale resolution of DNA methylation patterns, nor examine the relationship with

44 gene expression at the genome-wide level. Here, we provide the first high resolution methylome

45 of a mollusc and examine this in relationship to gene expression data to get a better

46 understanding of the role of DNA methylation in invertebrates.

\section{Methods}

48 Bisulfite treated DNA (BS-Seq) Analysis

49 The cohort of adult oysters used in this study was from Samish Bay, WA, USA. Briefly,

50 genomic DNA was isolated using DNAzol (Molecular Research Center) from gill tissue of 8

51 oysters, pooled, and methylation enrichment performed using the MethylMiner Kit (Invitrogen)

52 following the manufacturer's instructions. Specifically, pooled DNA was sheared by sonication

53 on a Covaris S2 (Covaris) (parameters: 10 cycles at 60 seconds each, duty cycle of $10 \%$, intensity

54 of 5, 100 cycles/burst). Approximately 13 ug of sheared DNA was used as input DNA and

55 incubated with MBD-Biotin Protein coupled to M-280 Streptavidin Dynabeads following the 
manufacturer's instructions (MethylMiner (Invitrogen)). Enriched, methylated DNA was eluted

57 from the bead complex with $1 \mathrm{M} \mathrm{NaCl}$ and purified by ethanol precipitation. This enriched

58 fraction represented approximately $15 \%$ of the total DNA recovered from the enrichment

59 procedure. The DNA library was prepared using the Illumina Tru-Seq system with methylated

60 TruSeq adapters (mean fragment size of library: $350 \mathrm{bp}$ ). Bisulfite treatment was then performed

61 using the EpiTect Bisulfite Kit (Qiagen) following manufacturer instructions. Library preparation

62 and sequencing was performed on the Illumina HiSeq 2000 platform at the University of

63 Washington high throughput sequencing facility (Seattle, WA). High-throughput reads (36bp

64 single end) were mapped back to the oyster genome (Fang et al., 2012) using BSMAP software

65 version 2.73 (Xi \& Li 2009). Methylation ratios (i.e. number of unconverted cytosines/the

66 number of converted and unconverted cytosines at each locus) were extracted from BSMAP

67 output (SAM) using a Python script (methratio.py) that is distributed with the BSMAP package.

68 Only cytosines in a $\mathrm{CpG}$ context with sufficient sequencing depth (defined here as greater than or

69 equal to $5 \mathrm{x}$ coverage) were retained for further analysis.

70

\section{DNA Methylation Landscape of Genomic Features}

In order to examine the relationship of DNA methylation and other genomic features, data from BSMAP (i.e. methratio) was converted to genomic feature tracks (i.e. generic feature format [GFF] files). Conversion was done using SQLShare (Howe et al., 2011), with the files and corresponding query language published (Gavery \& Roberts 2013).

The distribution of methylated CpGs with respect to specific genomic features was determined using BEDtools (i.e. intersectBED) (Quinlan \& Hall 2010). For this analysis, a CpG locus was considered methylated if at least half of the reads remained unconverted after bisulfite treatment. Genomic features that were examined include: exons and introns (Fang et al., 2012), 
79 promoters (defined as $1 \mathrm{~kb}$ upstream of open reading frames), and transposable elements. Putative

80 transposable elements were identified using RepeatMasker (Smit et al., 1996-2010), based on

81 protein similarities to the Transposable Element Protein Database. At the time of analysis the

82 database contained 5411 predicted proteins. For comparative purposes, total $\mathrm{CpG}$ across the

83 entire $C$. gigas genome was also examined. Locations of all CpGs were identified using the

84 EMBOSS tool fuzznuc (Rice et al., 2000), and the proportion of total CpG in each of the genomic

85 features listed above was determined using intersectBED. A Chi-squared test was performed to

86 determine if the distribution of methylated $\mathrm{CpG}$ was different from what would be expected by a

87 random distribution of the total $\mathrm{CpG}$ in the genome ( $\mathrm{p}$-value $<0.05$ was considered significant).

88 Average methylation ratios were determined for full length genes and also the cumulative

89 exons and cumulative introns comprising a gene. Average methylation was determined by the

90 number of methylated cytosines divided by the total number of $\mathrm{CpG}$ per region. Correlations

91 between the methylation status of exons and introns of individual genes were performed using

92 Pearson's correlation coefficient in SPSS (SPSS Inc.).

93 The relationship between predicted methylation status, using the CpG observed to

94 expected ratio ( $\mathrm{CpGo/e})$, and the average methylation ratio for each gene was examined to assess

95 the effectiveness of the $\mathrm{CpGo/e}$ method for predicting methylation in bivalve species. For this

96 analysis, the $\mathrm{CpGo/e}$ ratio was calculated for each gene using the method described in Gavery \&

97 Roberts (2010). Correlation between $\mathrm{CpGo} / \mathrm{e}$ and the methylation ratio was performed using

98 Spearman rank correlation in SPSS (SPSS Inc.).

99 Gene Expression Analysis

100 RNA was isolated from gill tissue of the same 8 individuals used for DNA isolation and

101 bisulfite sequencing using Tri-Reagent (Molecular Research Center). RNA was pooled in equal 
102 quantities and enriched for mRNA using Sera-Mag oligo dT beads (Thermo Scientific). First

103 strand synthesis was performed using SuperScript III (Invitrogen) and the second strand of cDNA

104 was synthesized using dUTP instead of dTTP, making the library strand-specific. A shotgun

105 library was constructed from double stranded cDNA for paired end sequencing by end-polishing,

106 A-tailing and ligation of sequencing adaptors. Sequencing was performed on the Illumina HiSeq

1072000 platform at the Northwest Genomics Center at the University of Washington (Seattle, WA).

108 High-throughput reads (50bp paired end) were mapped back to the oyster genome (Fang et al.,

109 2012) using CLC Genomics Workbench version 6.5 (CLC Bio). Initially, sequences were trimmed

110 based on quality scores of 0.05 (Phred, Ewing and Green, 1998; Ewing et al., 1998), and the

111 number of ambiguous nucleotides ( $>2$ on ends). Sequences smaller than $20 \mathrm{bp}$ were also

112 removed. For RNA-Seq analysis, expression values were measured as RPKM (reads per kilobase

113 of exon model per million mapped reads) (Mortazavi et al., 2008) with an unspecific match limit

114 of 10 and maximum number of 2 mismatches.

The RPKM values were used to examine the relationship between gene expression and

DNA methylation in gill tissue. All genes containing at least $1 \mathrm{CpG}$ locus $(\mathrm{n}=28,105)$ were

117 grouped into deciles according to transcriptomic representation in gill tissue (RPKM) and the

118 average methylation ratios for each decile were compared. A one-way ANOVA followed by

119 Tukey's test for multiple comparisons was performed using R (R Core Development Team 2012)

120 and a significance level of $\mathrm{p}<0.05$ was accepted.

121 A principal component analysis (PCA) was used as an exploratory tool to identify

122 relationships between DNA methylation, gene expression profiles and gene attributes such as

123 length. To explore variables related to gene expression, publicly available RNA-seq data from a

124 variety of adult $C$. gigas tissues were leveraged from Zhang et al. (2012). Specifically, mean

125 transcript abundance and variation in transcript abundance across tissues were calculated using 
126 RPKM values for 7 tissues adult tissues (digestive gland, female and male gonad, gill, anterior

127 muscle, hemocytes and labial palps). Mean transcript abundance was calculated using the mean

128 RPKM across all tissues for each gene. Variation in transcript abundance across tissues was

129 calculated as the coefficient of variation $(\% \mathrm{CV})$ of the RPKM across all 7 tissues for each gene.

130 Other gene attributes that were examined as they may associate with DNA methylation include

131 gene length, number of exons per gene and number of $\mathrm{CpG}$ per gene. In summary, the following

132 attributes were included as variables in the PCA performed in R (R Core Development Team

133 2012): average methylation ratio of the full length gene (as described above), gene length in base

134 pairs (bp), number of exons, average transcriptomic representation (average RPKM across 7 adult

135 tissues), coefficient of variation ( $\% \mathrm{CV})$ of transcript abundance (RPKM) among tissues. All

136 variables were log transformed, with the exception of the methylation ratio which was arcsine

137 transformed prior to analysis. The significance of each principal component was calculated using

138 Monte-Carlo randomization tests. Principal components were considered significant at $\mathrm{p} \leq 0.05$.

139 Correlation loadings of 0.6 were considered significant.

140 Results

141 DNA Methylation Mapping

142 Bisulfite treated DNA sequence reads $(139,728,554$ total reads; $36 \mathrm{bp})$ are available in the

143 NCBI Short Read Archive under the accession number SRX32737. A total of 120,734,949 reads

144 (86\%) mapped to the $C$. gigas genome. Fifty-six percent of the $164,873,219$ cytosines in the $C$.

145 gigas genome, had at minimum of $1 \mathrm{x}$ coverage. Of the 9,978,551 $\mathrm{CpG}$ dinucleotides in the

146 genome, 2.6 million $(26 \%)$ had $\geq 5 x$ coverage. The distribution of methylation ratios found at

$147 \mathrm{CpG}$ dinucleotides ranged between 0.0 - 1.0, but a majority of the loci were either heavily

148 methylated or unmethylated. Specifically, $55 \%(1,453,752)$ were methylated (i.e. $\geq 0.50)$ and 
another $28 \%$ were unmethylated (i.e. $=0.0)($ Figure 1). Genome feature track files (i.e. GFF)

150 representing 1) all $\mathrm{CpG}$ dinucleotides and 2) methylated $\mathrm{CpG}$ dinucleotides ( $>50 \%)$ for this

151 dataset were developed and are available (Gavery \& Roberts 2013).

\section{Methylation Landscape of Genomic Features}

153 Methylated $\mathrm{CpG}$ dinucleotides, defined as having a methylation ratio of 0.5 or greater,

154 were located predominantly in intragenic regions (exons and introns), but were also present in

155 putative promoters (defined as $1 \mathrm{~kb}$ upstream of TSS), transposable elements and unannotated

156 intergenic regions. The distribution of methylated $\mathrm{CpGs}$ across various genomic regions is

157 significantly different than what would be expected if the methylation were distributed randomly

158 throughout the $\mathrm{CpG}$ dinucleotides in the genome $\left(\mathrm{X}^{2}=513,194.1, \mathrm{df}=4, \mathrm{p}<0.0001\right)$.

159 Specifically, DNA methylation appears to be overrepresented in intragenic regions (64\% of

160 methylated $\mathrm{CpG}$ in combined exons and introns) when compared to the proportion of all $\mathrm{CpG}$ in

161 the genome (38\%) (Figure 2). When methylation was examined on a per gene basis a strong

162 positive correlation $\left(\mathrm{R}^{2}: 0.86\right)$ was observed between exonic and intronic methylation within a

163 gene. Additionally, a strong correlation was observed between the gene methylation measured via

164 high-throughput bisulfite sequencing and the predicted methylation ratio based on the $\mathrm{CpG}$

165 observed to expected ratio (CpGo/e) (Spearman rho: -0.616 , p-value: $\left.<1 \times 10^{-4}\right)$.

166 Gene Expression \& DNA methylation

167 After quality trimming, 45,751,574 RNA-seq reads mapped to the genome. Raw reads are

168 available in the NCBI Short Read Archive under the accession number TBD.

169 The relationship between the proportion of methylation in a gene and overall transcript

170 abundance in gill tissue was characterized (Figure 3). In general, transcription abundance 
171 increases significantly with increasing DNA methylation until the $40^{\text {th }}$ percentile after which it

172 remains relatively stable until the $100^{\text {th }}$ percentile when methylation significantly decreases.

173 The first two principal components (PC) of the PCA of gene attributes were significant

174 and explained $76.4 \%$ of the variation among the genes. This variation was being driven by

175 multiple factors, including DNA methylation (Figure 4 and Table 1). The only variable that did

176 not contribute significantly to the first two principal components was mean transcript abundance

177 (correlation loading 0.2). The first PC, which explained 50.2\% of the variation was loaded

178 heavily by number of $\mathrm{CpG}$ dinucleotides, the length of the mRNA and the number of exons. The

179 second $\mathrm{PC}$, which explained $26.1 \%$ of the variation was loaded heavily by the $\% \mathrm{CV}$ of gene

180 expression among tissues and the methylation ratio. DNA methylation is negatively correlated

181 with transcript variance between tissues $(\% \mathrm{CV})$ and relatively uncorrelated with attributes such

182 as gene length.

\section{Discussion}

184 Here we have used methylation enriched high-throughput bisulfite sequencing in

185 conjunction with genomic feature annotation and transcriptomic data in an attempt to gain a

186 better understanding of the role of DNA methylation in oysters. This work not only provides new

187 information on DNA methylation in invertebrates but also provides a framework for

188 characterizing DNA methylation in other taxa.

189 The reduced representation approach was selected to obtain a higher coverage of

190 methylated regions. In addition, since methylation was likely to occur in gene bodies (Zemach

191 2010), and because transcriptomic data was the primary genomic resource for C. gigas at the time

192 of sequencing (the genome was released soon after), it was expected that the methylation

193 enrichment would significantly limit the proportion of unmappable reads. Quantitative 
194 methylation data were obtained for both methylated $\mathrm{CpG}$ as well as unmethylated $\mathrm{CpG}$ that were

195 either interspersed with or flanking these more heavily methylated regions. Therefore,

196 methylation enriched bisulfite-sequencing was effective in generating a comprehensive

197 invertebrate methylome.

198 One of our primary findings was the overall level of genome methylation in the oyster.

199 Here we found that $15 \%$ of $\mathrm{CpG}$ dinucleotides ( $2 \%$ of total cytosines) are methylated in gill

200 tissue. This degree of methylation is much lower than the global methylation patterns seen in

201 mammals where 70-80\% of CpGs are methylated (Bird \& Taggart 1980), but still higher than

202 what has been reported in other invertebrates. For instance, only $0.8 \%$ of the CpGs are

203 methylated in the brain of $A$. mellifera (Foret et al., 2010) and between $0-8 \%$ of CpGs are

204 methylated in the nematode, Trichinella spiralis, depending on the life stage (Gao et al., 2012).

205 Although methylation in C. gigas is relatively high for an invertebrate, it is not outside the range

206 of what has been reported in other species by liquid chromatography-mass spectrometry analysis.

207 For example, similar to the oyster, $2 \%$ of total cytosines are methylated in the mollusc

208 Biomphalaria glabrata (Fneich et al.,2013). It should be noted that methylation in oysters does

209 likely vary in both a temporal and possibly tissue specific manner, as clearly indicated by Riviere

210 et al. (2013) by characterizing differences in total methylation during development. In addition,

211 because the sample represents a pool of multiple individuals, it cannot be determined whether the

212 variation in methylation at a particular locus represents hemimethylation or differential

213 methylation between individuals. In general, the bimodal pattern observed (Figure 1) indicates

214 that a $\mathrm{CpG}$ locus is either heavily methylated or unmethylated, but future work sequencing

215 individual oysters would provide valuable information regarding individual epigenetic variation

216 in oysters. 
This work also provided the first direct evidence in oysters that DNA methylation is

218 prominent in gene bodies (see Figure 2) and these data are well correlated with previous

219 investigations using an in silico approach (i.e. CpGo/e) to predict methylation in C. gigas

220 (Gavery \& Roberts 2010). The predominance of gene body methylation is consistent with what

221 has been described in other invertebrates (e.g. Suzuki et al., 2007, Zemach et al., 2010, Lyko et

222 al., 2010) and there is increasing evidence that gene body methylation is the ancestral pattern

223 (Lechner et al., 2013). The function of gene body methylation remains unclear, but studies

224 indicate possible active roles in preventing spurious transcription (Bird et al.,1995, Huh et

225 al.,2013) and regulating alternative splicing (Manukea et al., 2010, Shukla et al., 2011, Foret et

226 al., 2012), as well as a more passive role for methylation as a byproduct of an open chromatin

227 state (Jjingo et al., 2012). Given the nature of the study design, we are not able to directly test the

228 hypothesis that DNA methylation contributes to spurious transcription or the regulation of

229 alternative isoforms in C.gigas. However, genomic feature tracks have been developed and

230 published (Gavery \& Roberts 2013) so that genome wide methylation can be easily visualized

231 with respect to gene expression patterns (exon-specific RPKM).

232 Exons are the preferential target for gene body methylation for most species (Feng et al.,

233 2012), and methylation was enriched in exons in the oyster. However, there is also a relatively

234 large amount of intronic methylation in oysters when compared to other invertebrate species. For

235 example, DNA methylation occurs almost exclusively in exons in the honey bee $A$. mellifera

236 (Lyko et al., 2010). Genome-wide methylation studies in other invertebrate species also report

237 very low levels of intronic methylation relative to other genomic regions (e.g. Gao et al, 2012,

238 Bonasio et al., 2012). Similarly, in plants, methylation is preferably targeted to exons; however it

239 has been reported that in globally methylated mammalian genomes gene body methylation is not

240 biased toward exons (Feng et al., 2012), although exon/intron boundaries can be marked by 
241 differences in DNA methylation (Sati et al., 2012). It appears that bivalves may be unique among

242 the invertebrates examined in terms of the degree of methylation in introns. Intronic methylation

243 has been implicated to be involved in gene regulation through the expression of alternative

244 isoforms of genes in other species (e.g. Manukea et al., 2010, Foret et al., 2012). Variation in

245 methylation patterns between taxa may indicate that additional model invertebrates are needed to

246 study the function of these epigenetic marks.

247 This distribution of DNA methylation in the C. gigas genome is consistent with the

248 fractionated or 'mosaic' pattern of methylation previously described in invertebrates (Tweedie et

249 al., 1999, Simmen et al 1999). In oysters, as in other invertebrates, the methylated fraction tends

250 to consist of gene bodies, while other genomic regions exhibit less methylation (Figure 2).

251 Interestingly, transposable elements (TE) show little methylation in oyster gill tissue. This is in

252 contrast to vertebrate genomes where TE are heavily methylated and function to suppress their

253 activity (Yoder et al 1997). While there is no general consensus regarding the extent of TE

254 methylation across invertebrate taxa, the pattern of sparse TE methylation observed in oysters is

255 similar to what has been described in other invertebrate species (Simmen et al 1999, Feng et al

256 2010, Zemach et al 2010).

257 Intra-genic DNA methylation is positively correlated with gene expression in C. gigas

258 with the moderately and highly expressed genes showing the highest degree of methylation

259 (Figure 3). This relationship is similar to what has been reported for other invertebrate species

260 (Zemach et al., 2010). Interestingly, Riviere et al (2013) reported a negative relationship between

261 DNA methylation and expression of certain homeobox (hox) genes during embryonic

262 development in C. gigas. The authors hypothesized that the apparent suppression of hox

263 expression by DNA methylation may be due to repression by DNA methylation proximal to the

264 transcription start site in these genes. Although the results reported here and those of Riviere et al. 
may appear contradictory, it is possible that depending on the context of the methylation (i.e.

266 whether gene body or promoter methylation) it may play either a repressive or expressive role.

267 This is referred to as the DNA methylation paradox (Jones 1999) and is observed in a wide range 268 of taxa.

269 We used an ordination approach to explore genomic attributes or groups of attributes that

270 predictably co-occur with methylated genes in the C. gigas genome. Because multiple factors

271 may be linked with methylation (either through causative or correlative associations), this

272 approach allowed us to identify relationships between multiple variables. The most interesting

273 finding from the PCA analysis is that amount of methylation in a gene is related to the variance in 274 expression between tissues. Genes that show the least variation in expression between tissues

275 have higher DNA methylation levels than those exhibiting a tissue-specific expression profile

276 (i.e. high $\% \mathrm{CV}$ between tissues). This observation provides corroboration for previous reports

277 based on in silico analyses in oysters showing that housekeeping genes have the highest amount

278 of methylation in C. gigas (Gavery \& Roberts 2010). Housekeeping genes perform functions

279 required by all cell types, therefore it's expected that their expression patterns would show low

280 variation across tissues. The results of this study are consistent with that expectation that genes

281 with low expression variation across tissues show a high degree of methylation relative to genes

282 with a more tissue-specific expression pattern. Again, this study supports previous findings

283 (Gavery \& Roberts 2010, Roberts \& Gavery 2012) that heavily methylated genes are enriched in

284 housekeeping functions, which are essential for cellular function. One theory is that the lack of

285 methylation in these genes with more tissue-specific expression is that it can contribute to

286 phenotypic plasticity by allowing more transcriptional opportunities through process such as

287 allowing access to alternative TSS, facilitate exon skipping or other alternative splicing

288 mechanisms and allow for increased sequencing variation (Roberts \& Gavery 2012). 


\section{Conclusions}

290

291

292

293

294

295

296

297

298

299

300

301

302

303

304

305

306

307

308

309

310

Through the current effort, quantitative methylation data were obtained for over 2.5 million CpG dinucleotides throughout the genome of Crassostrea gigas. These data represent the first high resolution methylome in any mollusc and the analytical approaches provide a framework for DNA methylation characterization in other species. In addition, this dataset developed here will be beneficial for phylogenetic analysis of DNA methylation in invertebrates, which will be more robust with the addition of a lophotrochozoan species. The results of this study highlight similarities in epigenetic profiles of invertebrates such as a predominance of gene body methylation and a positive relationship between intragenic methylation and gene expression. In addition, they highlight interesting differences between invertebrate epigenomes including a higher level of intronic methylation in bivalves than what has been reported, for example, in insects. Although the functional role of DNA methylation in bivalves remains elusive, two scenarios could explain our findings. One possibility is DNA methylation in gene bodies is a byproduct of transcription resulting from an open chromatin state, as proposed by Jjingo et al (2013). Thus the methylation patterns are influenced by transcriptional activity. The second scenario is DNA methylation is involved in regulating the gene activity in bivalves. If in fact DNA methylation does influence transcription, the regulatory role is likely very complex. For instance, DNA methylation could have both a have direct regulatory effect on certain genes as proposed by Riviere et al. (2013), as well as facilitating expanded transcriptional opportunities in other cases. Future studies will certainly be challenging given the dynamic nature of DNA methylation, but will hopefully help better delineate if DNA methylation plays a functional role in regulating genome activity in bivalves and what that role might be.

\section{Acknowledgements}


312 The authors would like to thank Bill Howe and Daniel Halperin for their assistance with

313 SQLShare. They would also like to acknowledge Samuel J White for comments that improved 314 the manuscript.

\section{References}

316 Bell AC, Felsenfeld G. 2000. Methylation of a CTCF-dependent boundary controls imprinted 317 expression of the Igf2 gene. Nature 405:482-5.

318 Bird A. 1995. Gene number, noise reduction and biological complexity. Trends Genet 11:94-100.

319 Bird AP, Taggart MH.1980. Variable patterns of total DNA and rDNA methylation in animals.

320 Nucleic Acid Res 8:1485-1497.

321 Bonasio R, Li Q, Lian J, MuttiNS, Jin L, Zhao H, Zhang, P, Wen P, Xiang H, DingY, Jin Z, Shen

322 S, Wang Z, Wang W, Wang J, Berger S, Liebig J, Zhang G. 2012. Genome-wide and caste-

323 specific DNA methylomes of the ants Camponotus floridanus and Harpegnathos saltator. Curr

324 Biol 22:1755-1764.

325 Colot V, Rossignol JL.1999. Eukaryotic DNA methylation as an evolutionary

326 device. BioEssays 21:402-411.

327 Fang X, Li L, Luo R, Xu F, Wang X, Zhu Y, Yang L, Huang Z. 2012. Genomic data from the

328 Pacific oyster (Crassostrea gigas). GigaScience. http://dx.doi.org/10.5524/100030.

329 Feng S, Cokus SJ, Zhang X, Chen PY, Bostick M, Goll MG, Hetzel J, Jain J,Strauss SH, Halpern

330 ME, Ukomadu C, Sadler KC, Pradhan S, Pellegrini M, Jacobsen SE. 2010. Conservation and

331 divergence of methylation patterning in plants and animals. Proc Natl Acad Sci 107: 8689-8694. 
332 Fneich S, Dheilly N, Adema C, Rognon A, Reichelt M, Bulla J, Grunau C, Cosseau C. 2013. 5-

333 methyl-cytosine and 5-hydroxy-methyl-cytosine in the Genome of Biomphalaria Glabrata, a Snail

334 Intermediate Host of Schistosoma Mansoni. Parasites \& Vectors 6:167.

335 Foret S, Kucharski R, Pellegrini M, Feng S, Jacobsen SE, Robinson GE, Maleszka R. 2012. DNA

336 Methylation Dynamics, Metabolic Fluxes, Gene Splicing, and Alternative Phenotypes in Honey

337 Bees. Proceedings of the National Academy of Sciences 109:4968-4973.

338 Gao F, Liu XS, Wu X-P, Wang X-L, Gong D, Lu H, Song Y, Wang J, Du J, Liu S, Han X, Tang Y,

339 Yang H, Jin Q, Zhang X, Liu M. 2012. Differential methylation in discrete developmental stages

340 of the parasitic nematode Trichinella spiralis. Genome Biol 13:R100

341 Gavery M, Roberts SB. 2010. DNA methylation patterns provide insight into epigenetic

342 regulation in the Pacific oyster (Crassostrea gigas). BMC Genomics 11:483.

343 Gavery M, Roberts S. 2013. Crassostrea gigas high-throughput bisulfite sequencing (gill tissue).

344 figshare. [http://dx.doi.org/10.6084/m9.figshare.749728] Retrieved 18:44, Oct 21, 2013 (GMT).

345 Howe B, Cole G, Souroush E, Koutris P, Key A, Khoussainova N, Battle L. 2011. Database-as-a-

346 service for long-tail science. In Proceedings of the 23rd international conference on Scientific and

347 statistical database management (SSDBM'11), Judith Bayard Cushing, James French, and Shawn

348 Bowers (Eds.). Springer-Verlag, Berlin, Heidelberg, 480-489.

349 Hsieh CL. 1994. Dependence of transcriptional repression on CpG methylation density. Mol Cell

350 Biol 14:5487-5494.

351 Huh I, Zeng J, Park T, Yi S. 2013. DNA Methylation and Transcriptional Noise. Epigenetics \& 352 Chromatin 6:9. 
353 Jjingo D, Conley AB, Yi SV, Lunyak VV, Jordan IK. 2012. On the presence and role of human

354 gene-body DNA methylation. Oncotarget 3:462-474.

355 Jones PA. 1999. The DNA Methylation Paradox. Trends in Genetics 15:34-37.

356 Kucharski R, Maleszka J, Foret S, Maleszka R. 2008. Nutritional Control of Reproductive Status

357 in Honeybees via DNA Methylation. Science 319:1827-1830.

358 Lechner M, Marz M, Ihling C, Sinz A, Stadler PF, Krauss V. 2013. The Correlation of Genome

359 Size and DNA Methylation Rate in Metazoans. Theory in Biosciences 132:47-60.

360 Li E, Bestor TH, Jaenisch R. 1992. Targeted mutation of the DNA methyltransferase gene results

361 in embryonic lethality. Cell 69:915-926.

362 Lyko F, Foret S, Kucharski R, Wolf S, Falckenhayn C, Maleszka R. 2010. The Honey Bee 363 Epigenomes: Differential Methylation of Brain DNA in Queens and Workers. PLoS Biology 364 8:e1000506.

365 Maunakea AK, Nagarajan RP, Bilenky M, Ballinger TJ, D’Souza C, Fouse SD, Johnson BE,

366 Hang C, Nielsen C, Zhao Y, Turecki G, Delaney A, Varhol R, Thiessen N, Shchors K, Heine VM,

367 Rowitch DH, Xing X, Fiore C, Schillebeeckx M, Jones SJM, Haussler D, Marra MA, Hirst M,

368 Wang T, Costello JF. 2010. Conserved Role of Intragenic DNA Methylation in Regulating

369 Alternative Promoters. Nature 466:253-257.

370 Okano M, Bell DW, Haber DA, Li E. 1999. DNA methyltransferases Dnmt3a and Dnmt3b are

371 essential for de novo methylation and mammalian development. Cell 99:247-257.

372 Petrovic V, Perez-Garcia C, Pasantes JJ, Satovic E, Prats E, Plohl M. 2009. A GC-rich Satellite

373 DNA and Karyology of the Bivalve Mollusk Donax Trunculus: a Dominance of GC-rich

374 Heterochromatin. Cytogenet Genome Res 124: 63-71. 
375 Quinlan AR, Hall IM. 2010. BEDTools: a flexible suite of utilities for comparing genomic

376 features. Bioinformatics 26:841-842.

377 R Development Core Team: R: A language and environment for statistical computing.

378 [http://www.R-project.org].

379 Rice P, Longden I, Bleasby A. 2000. EMBOSS: the European Molecular Biology Open Software 380 Suite. Trends Genet 16:276-7.

381 Riviere G, Wu GC, Fellous A, Goux D, Sourdaine P, Favrel P. 2013. DNA Methylation Is Crucial

382 for the Early Development in the Oyster C. gigas. Marine Biotechnology 1-15.

383 doi:10.1007/s10126-013-9523-2.

384 Roberts SB, Gavery MR. 2012. Is There a Relationship Between DNA Methylation and

385 Phenotypic Plasticity in Invertebrates? Frontiers in Physiology 2:116.

386 Sati S, Tanwar VS, Kumar KA, Patowary A, Jain V, Ghosh S, Ahmad S, Singh M, Reddy SU,

387 Chandak GR, Raghunath M, Sivasubbu S, Chakraborty K, Scaria V, Sengupta S. 2012. High

388 resolution methylome map of rat indicates role of intragenic DNA methylation in identification of

389 coding region. PloS One 7:e31621.

390 Shukla S, Kavak E, Gregory M, Imashimizu M, Shutinoski B, Kashlev M, Oberdoerffer P,

391 Sandberg R, Oberdoerffer S. 2011. CTCF-promoted RNA Polymerase II Pausing Links DNA

392 Methylation to Splicing. Nature 479:74-79.

393 Simmen MW Leitgeb S, Charlton J, Jones SJM, Harris BR, Clark VH, Bird A. 1999.

394 Nonmethylated transposable elements and methylated genes in a chordate genome. Science $395 \quad 283: 1164-1167$. 
396 Smit AFA, Hubley R, Green P. 1996-2010. RepeatMasker Open-3.0.

397 [http://www.repeatmasker.org].

398 Suzuki MM, Kerr ARW, De Sousa D, Bird A. 2007. CpG methylation is targeted to transcription 399 units in an invertebrate genome. Genome Res 17:625-631.

400 Tweedie S, Charlton J, Clark V, Bird A. 1999. Methylation of genomes and genes at the 401 invertebrate-vertebrate boundary. Mol Cell Biol 17:1469-1475.

402 Wang S, Bao Z, Hu X, Shao M, Zhang L, Hu J. 2008. Two Novel Elements (CFG1 and PYG1) of 403 Mag Lineage of Ty3/Gypsy Retrotransposons from Zhikong Scallop (Chlamys Farreri) and 404 Japanese Scallop (Patinopecten Yessoensis). Genetica 133:37-46.

405 Wolffe AP, Matzke MA. 1999. Epigenetics: regulation through repression. Science 286:481-486.

406 Xi Y, Li W. 2009. BSMAP: whole genome Bisulfite Sequence MAPping program. BMC 407 Bioinformatics 10:232.

408 Yoder JA, Walsh CP, Bestor TH. 1997. Cytosine Methylation and the Ecology of Intragenomic 409 Parasites. Trends in Genet 8:335-340.

410 Zemach A, McDaniel IE, Silva P, Zilberman D. 2010. Genome-Wide Evolutionary Analysis of 411 Eukaryotic DNA Methylation. Science 328:916-919.

412 Zhang G, Fang X, Guo X, Li L, Luo R, Xu F, Yang P, Zhang L, Wang X, Qi H, Xiong Z, Que H, 413 Xie Y, Holland PWH, Paps J, Zhu Y, Wu F, Chen Y, Wang J, Peng C, Meng J, Yang L, Liu J, Wen 414 B, Zhang N. 2012. The oyster genome reveals stress adaptation and complexity of shell 415 formation. Nature 490:49-54. 
416 Zhang X, Yazaki J, Sundaresan A, Cokus S, Chan SW, Chen H, Henderson IR, Shinn P, Pellegrini

417 M, Jacobsen SE, Ecker JR. 2006. Genome-wide high-resolution mapping and functional analysis

418 of DNA methylation in Arabidopsis. Cell 126:1189-1201.

419 Zilberman D, Gehring M, Tran RK, Ballinger T, Henikoff S. 2007. Genome-wide analysis of

420 Arabidopsis thaliana DNA methylation uncovers an interdependence between methylation and

421 transcription. Nat Genet 39: 61-69. 


\section{Figure 1}

Frequency distribution of methylation ratios for $\mathrm{CpG}$ dinucleotides in oyster gill tissue

A total of 2,625,745 CpG dinucleotides with $\geq 5 x$ coverage are represented.

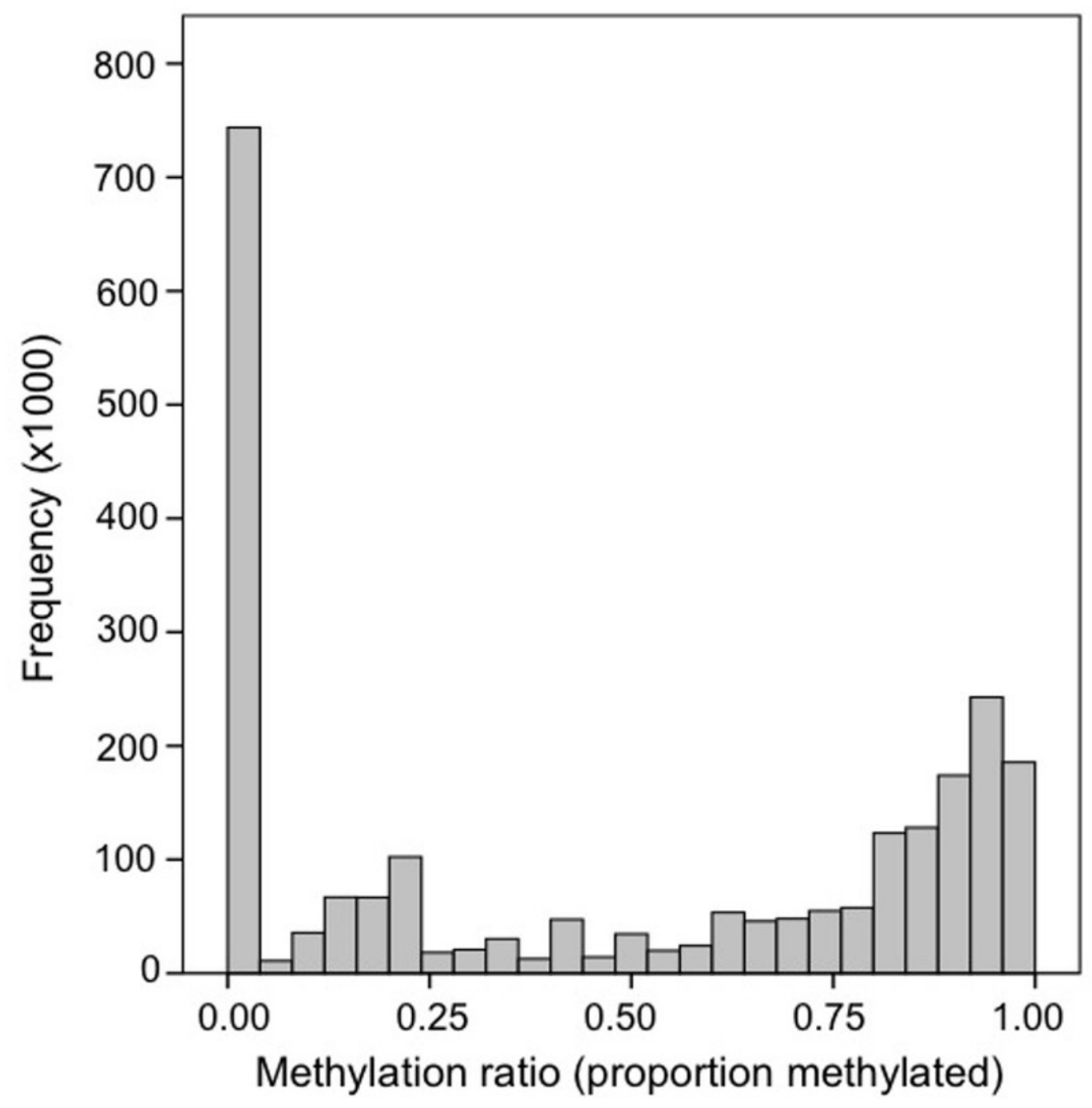




\section{Figure 2}

Comparison of the total $\mathrm{CpG}$ versus methylated $\mathrm{CpG}$ in oyster gill tissue by genomic feature

Proportion of all $\mathrm{CpG}$ (blue) and methylated $\mathrm{CpG}$ (red) in gill tissue across genomic features of C. gigas. Percent of CpG dinucleotides in Exons, Introns, Transposable Elements (TE), promoters (Pro) and unannotated intergenic regions (Other) are reported






\section{Figure 3}

DNA methylation among genes with increasing transcript abundances.

Expressed genes were grouped into deciles by transcription abundance. Genes not expressed in gill (i.e. RPKM=0) are also shown (leftmost column).. Error bars represent 95\% confidence intervals.

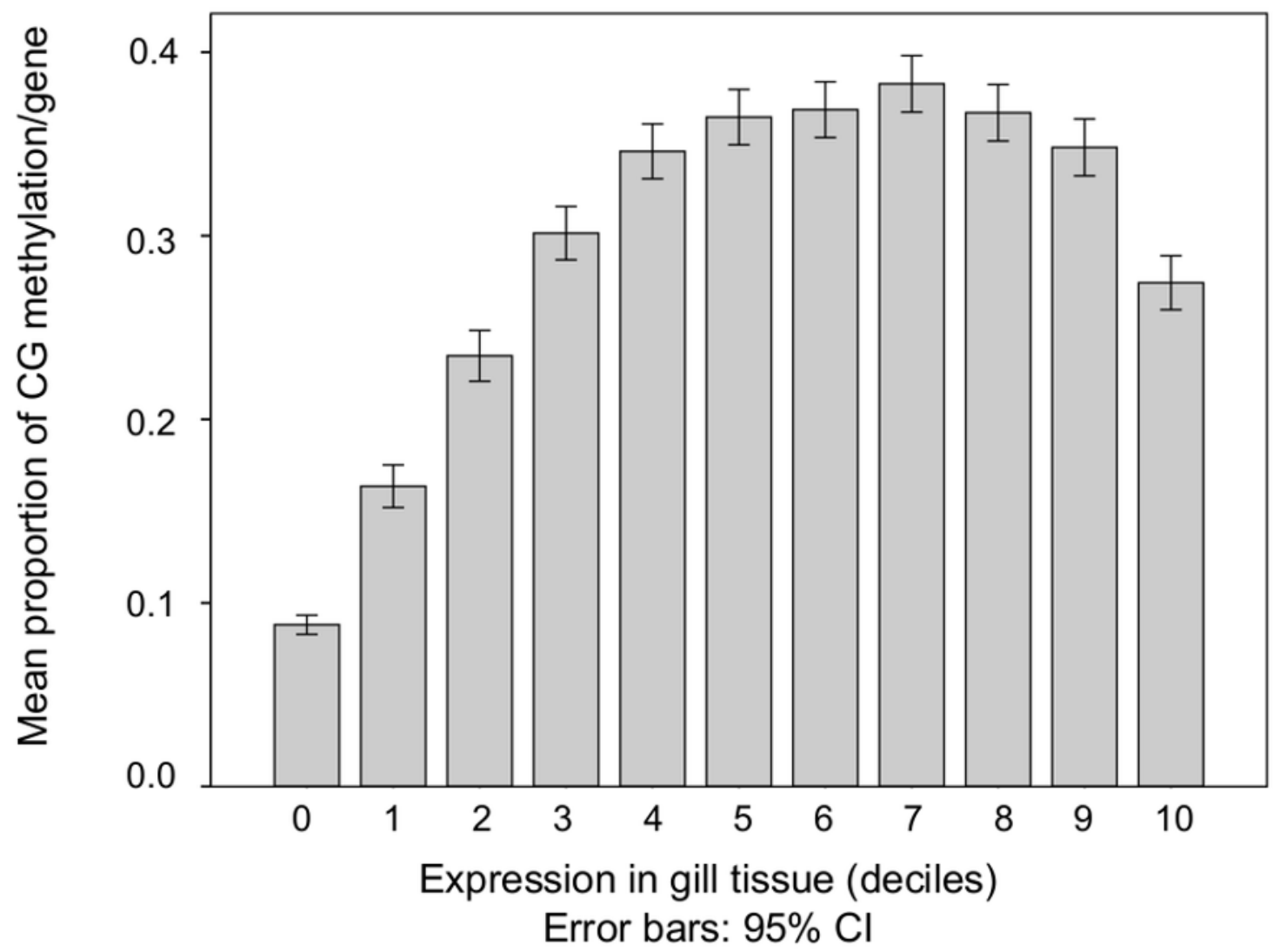




\section{Figure 4}

PCA ordination of oyster genes by gene attributes

Variables loadings shown by purple arrows. Variables significantly contributing to PC1 and PC2 include: methylation ratio (Methylation), the coefficient of variance of expression between tissues (\%CV), the number of exons (Exons), the length of the mRNA in base pairs (mRNA) and the number of $\mathrm{CpG}$ dinucleotides in the gene (CpG). Variables that did not significantly contributes to PC1 and PC2 include the mean transcript abundance (Expression). Inset depicts ordination of the genes analyzed on PC1 and PC2 ( $n=27,181)$

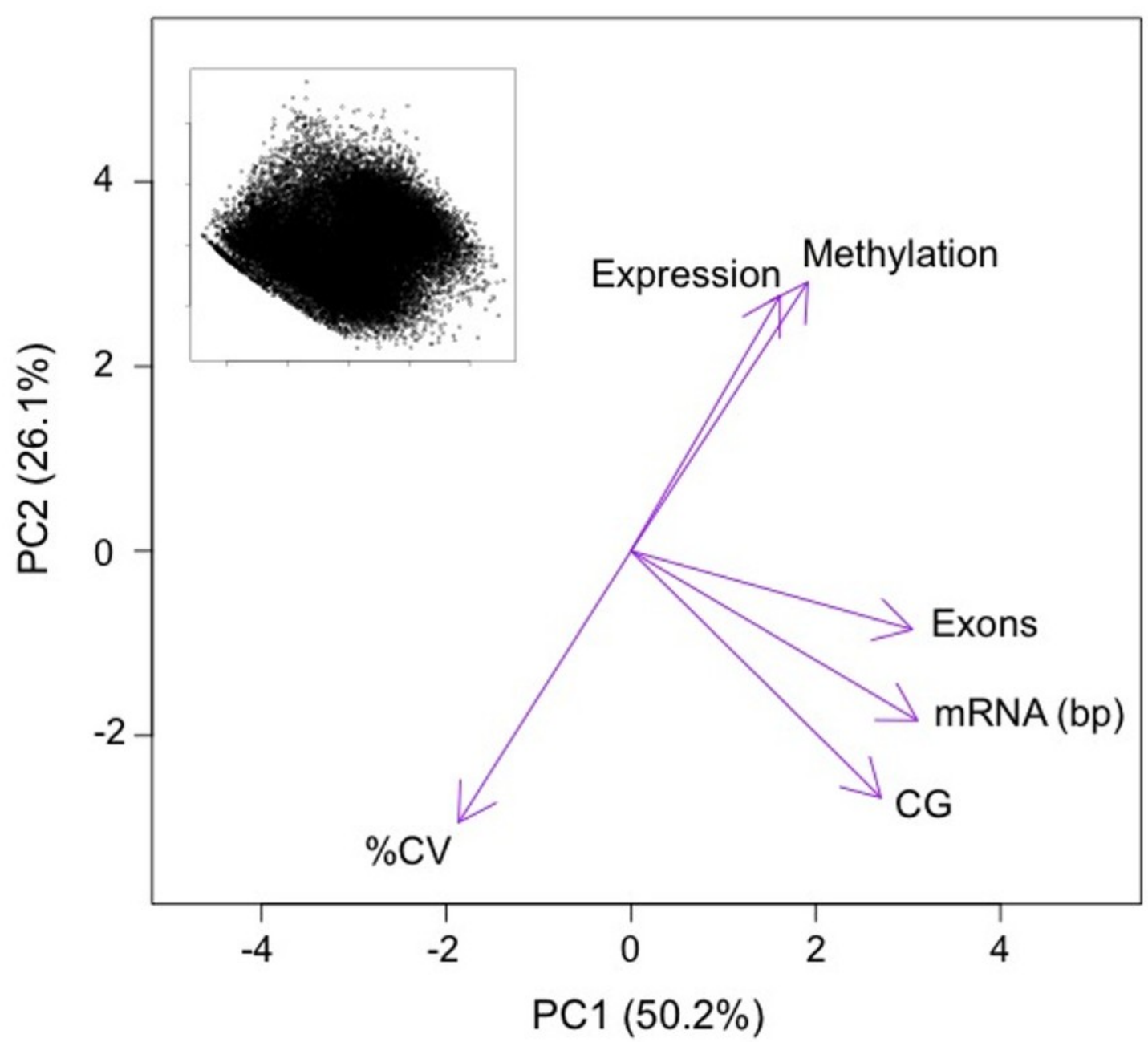




\section{Table 1 (on next page)}

Summary of PCA for gene attributes 


\begin{tabular}{ccclc}
\hline $\begin{array}{c}\text { Principal } \\
\text { Component }\end{array}$ & $\begin{array}{c}\text { \% variance } \\
\text { explained }\end{array}$ & Significance value & \multicolumn{2}{c}{$\begin{array}{c}\text { Significant variable } \\
\text { loadings }\end{array}$} \\
\hline \multirow{2}{*}{ PC1 } & \multirow{2}{*}{50.2} & & Number of CpG & 0.9 \\
& \multirow{2}{*}{26.1} & $<0.001$ & Length mRNA & 0.9 \\
\multirow{2}{*}{ PC2 } & & Number of exons & 0.8 \\
& \multirow{2}{*}{$* 0.0001$} & Expression \%CV & -0.6 \\
& & & Methylation ratio & 0.6 \\
\cline { 3 - 5 } & & & &
\end{tabular}

